\title{
220 m Altpleistozän im „Heidelberger Loch“
}

\author{
FRITZ FEZER*)
}

Pleistocene, fluvial sediments, stratigraphy, climatic change, Heidelberg, SW-Germany

\begin{abstract}
Kurzfassung: Am westlichen Ende seiner Steilstrecke hat der Neckar eine Art Binnendelta aufgeschüttet. Er hat sofort reagiert, wenn im nahen Odenwald-Bergland die Ökosysteme gestört oder zusammengebrochen waren. Wenn sie sich wieder eingespielt hatten, trafen Gerölle aus weiter entfernten Gesteinsprovinzen ein. Mit der Aufschüttung glich der Fluß die tektonische Absenkung des „Heidelberger Lochs" aus. Vergleichbare Profile aus Holland liegen näher am nordischen Klimabereich, und die Moränen und Schotterserien des Alpenvorlandes repräsentieren nur das Maximum einer Kaltzeit. Demgegenüber erlauben die 5 Profile aus Heidelberg in einer einzigartigen Vollständigkeit und ihren vielen Details, die geoökologischen Prozesse zu rekonstruieren, die im vom Eis wenig beeinträchtigten Teil Mitteleuropas abgelaufen sind.
\end{abstract}

\section{[220 $\mathrm{m}$ thick Old Pleistocene fluvial sediments in the Upper Rhine graben near Heidelberg]}

\begin{abstract}
The Neckar river passes the Odenwald mountains in a gorge (tectonic uplift) and flows in Heidelberg into the Upper Rhine graben, which sinks here by 0,2 to $0,75 \mathrm{~mm}$ year. In this deltalike voluminous sediment the whole Quarternary is preserved. In the Cold Ages braided rivers spread gravel and sand. In the interglacials a meandering Neckar sedimented loam, sand or thin layers of gravel. In the warm stages from 35 to 7 the curve of the clay content was tuned to the 180 -temperature curves of the oceans.
\end{abstract}

\section{[220 m Paléopléistocène à Heidelberg]}

Résumé: Le Neckar a constitué par son cône de déjections une sorte de delta intérieur à l'extrémité oú-est de la partie de son cours à forte déclivité. Il a immédiatement réagi, lorsque les systèmes écologiques du proche Odenwald-Bergland furent perturbés ou s'effondrèrent. Quand ceux-ci se furent reéquilibrés, des cailloutis s'accumulèrent, provenant de strates pierreuses de régions plus éloignées.

Par ce remblaiement, le fleuve compensa l'affaissement tectonique du Heidelberg Loch (trou de Heidelberg). En Hollande, des profils comparables sont plus à rapprocher des zones climatiques nordiques; en zones préalpines, les moraines et séries d'éboulis représentent le maximum d'une période froide. Par contre, les 5 profils de Heidelberg permettent de reconstituer, de façon unique l'intégralité et avec force détails, les processus géoécologiques qui se sont déroulés dans une partie de l'Europe centrale peu affectée par les glaces.

\section{Inhaltsverzeichnis}

1 Einleitung

2 Das Thermalprofil *) Anschrift des Verfassers: Prof. Dr. F. Fezer, Moselbrun-
nenweg 91, 69118 Heidelberg
3 Das Profil „HD 1Fenneberger“, klimastratigraphische Einpassung

4 Sedimentanalyse

5 Neckarsande in Heidelberg (300-285 m) und Mauer (132 bis $141 \mathrm{~m} \mathrm{NN}$ ), Kaltzeit 18 und Warmzeit 17

6 Ergebnisse und Ausblick

7 Dank

8 Schriftenverzeichnis

\section{Einleitung}

\begin{abstract}
„Preference should be given to a study of long records - those records which are more complete than others ... to solve the puzzle of Quarternary terrestrial stratigraphy" (Schlüchter 1992, 607).
\end{abstract}

In der Gegenwart sinkt der Untergrund in der Nähe des Heidelberger Thermalbads tektonisch ab, 0,8 - 1 $\mathrm{mm} / \mathrm{Jahr}$. Hier konnte der Neckar zu allen Zeiten sein Sediment ablagern, nur nach der Lone-Ablenkung von Plochingen (ca. 1 Mio - 0,8 Mio J.v.h.) hat er eine Zeit lang - wie viele andere Rhein-Nebenflüsse - sein Tal verschüttet (Fezer 1992, in BeinHauER \& WAGNER). Ansonsten konnte er rückwärts einschneiden und andere Flüsse zu sich ablenken.

Aus den Krümmungsradien hochgelegener alter Umlauftäler im Odenwald läßt sich das Einzugsgebiet des Neckars zu Beginn des Pleistozän schätzen.

\begin{tabular}{|c|c|c|c|c|}
\hline & Niveau über Fluß & Radius & Einzugsgebiet & Quelle \\
\hline $\begin{array}{l}\text { Beginn des } \\
\text { Pleistozäns }\end{array}$ & $+70 \mathrm{~m}$ & $375 \mathrm{~m}$ & $<2500 \mathrm{~km}^{2}$ & Pforzheim? \\
\hline heute & 0 & $700 \mathrm{~m}$ & $14000 \mathrm{~km}^{2}$ & $\begin{array}{l}\text { V.-Schwen- } \\
\text { ningen }\end{array}$ \\
\hline
\end{tabular}

Gegenüber den Gewinnen wiegen die kleinen Verluste an den Main leicht (zur Flußgeschichte s. Fezer 1974 und 1993/95, MADER 1978). Im Lauf des Pleistozäns hat er immer mehr und immer gröberes Material im „Heidelberger Loch“ abgelagert, woran die Klimaverschlechterung sowie ein schnelleres tektonisches Absinken beteiligt sind.

Insgesamt wurden 5 Bohrungen ausgewertet (Abb. 2). Selbstverständlich sind wir bei der stratigraphischen Interpretation der Profile von der Flur nach unten vorgegangen. Die jüngsten Schotter sind eini- 


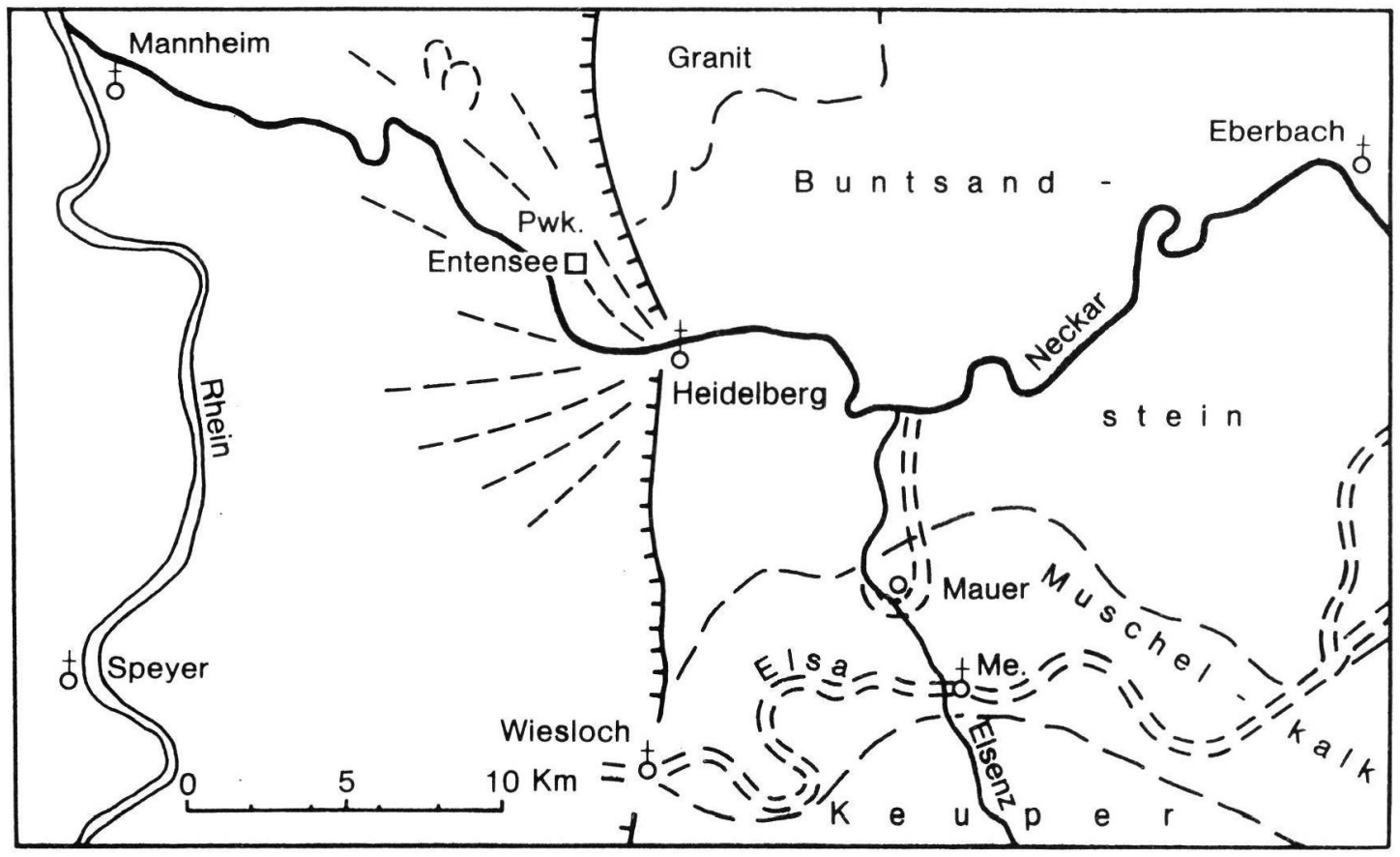

Abb. 1: Im Einzugsgebiet des Unteren Neckars anstehende Gesteine und sein Heidelberger Schwemmfächer. Elsa = hy pothetisches Tal aus der Tertiärzeit.

Fig. 1: Origin of different nearby pebbles. Elsa = supposed valley of Tertiary age.

germaßen einzuordnen. Ferner haben wir die Profile zunächst in konventioneller Weise gezeichnet. Später schritt, um das Verständnis der Prozessè zu fördern, die Beschreibung von den alten zu den jungen Schichten, von der Quartärbasis zum Ende des Altpleistozäns. Wie in den Naturwissenschaften üblich, läuft die Zeitachse der Diagramme von links nach rechts, und die Variablen sind an der Ordinate ausgerichtet; das Bohrprofil ist also um $90^{\circ} \mathrm{im}$ Uhrzeigersinn verschwenkt.

Das Neckargebiet lag in den Eiszeiten von den vergletscherten Flächen Mitteleuropas so weit weg, daß das Klima kaum sekundär beeinflußt, sondern nur vom Wandel des Erdklimas geprägt wurde. Deshalb lassen sich die Heidelberger Schichten gut mit der ozeanischen Stratigraphie korrelieren.

\section{Das Thermalprofil von 650 bis 390 m Teufe}

Auf Anregung von SALOMON (1927) wurde 1913-1918 beim jetzigen Thermalbad nach Warmwasser gesucht und zwischen 411 und $1022 \mathrm{~m}$ Teufe auch mehrere Thermen gefunden (Abb. 2). Weil sich von gelegentlich auftretenden Pflanzenresten nur die Familie bestimmen und die Gattung vermuten ließ, bauten die Geologen ihre Stratigraphie auf Ölspuren und Asphalt auf, die sie mit dem unterelsässischen Oligozän gleichsetzten. Die verschiedenen von SALOMON (1927) und BARTZ (1951) angenommenen
Grenzen zwischen Plio- und Pleistozän sind auf jeden Fall überholt, weil diese jetzt auf 2,4 Mio J.v.h angesetzt wird. Zu dieser Zeit erreicht erstmals Treibeis das Rockall-Plateau zwischen Island und Irland (SHACKLETON 1989, 11). Unbeeinflußt von solchen Überlegungen hat Bohrmeister Kößler aufgeschrieben, bei welcher Teufe er auf "weicheres" (Ton) und „härteres" Material (Sand) gestoßen ist. Eine Kladde, die von $387 \mathrm{~m}$ bis $495 \mathrm{~m}$ Teufe reicht, blieb im Universitätsarchiv neben anderen Aktenbündeln zur Thermalbohrung erhalten.

Übertragen wir die Stratigraphie aus den Bohrungen Entensee (s.u.) auf das etwas mächtigere Thermalprofil, dann wäre der Beginn des Pleistozäns in etwa 650 m Teufe anzunehmen. Unter den westlichen Stadtteilen von Heidelberg lagert also ca. 640 m Pleistozän. Von 650 bis $400 \mathrm{~m}$ Teufe haben Neckar und „Randfluß" (FEZER 1974) einen grauen bis graugelben, teils mergeligen, teils feinsandigen Ton, nur vereinzelt Gerölle abgelagert. Jene bestehen aus gebleichtem Buntsandstein, Quarz, Quarzit, Karneol, Kieselschiefer und Hornsteinen aus dem Muschelkalk. Nur bei $-600 \mathrm{~m}$ traten auch Kalkbröckchen hinzu. Die Zusammensetzung spricht für eine starke chemische und mechanische Verwitterung und für einen Fluß mit mäßigen Hochwässern. Nicht alle diese Gesteine stehen im südlichen Odenwald an, ein Teil stammt aus dem Schwarzwald (BARTZ 1951).

In 397 m Teufe fanden sich Kalkgeröllchen, und von 


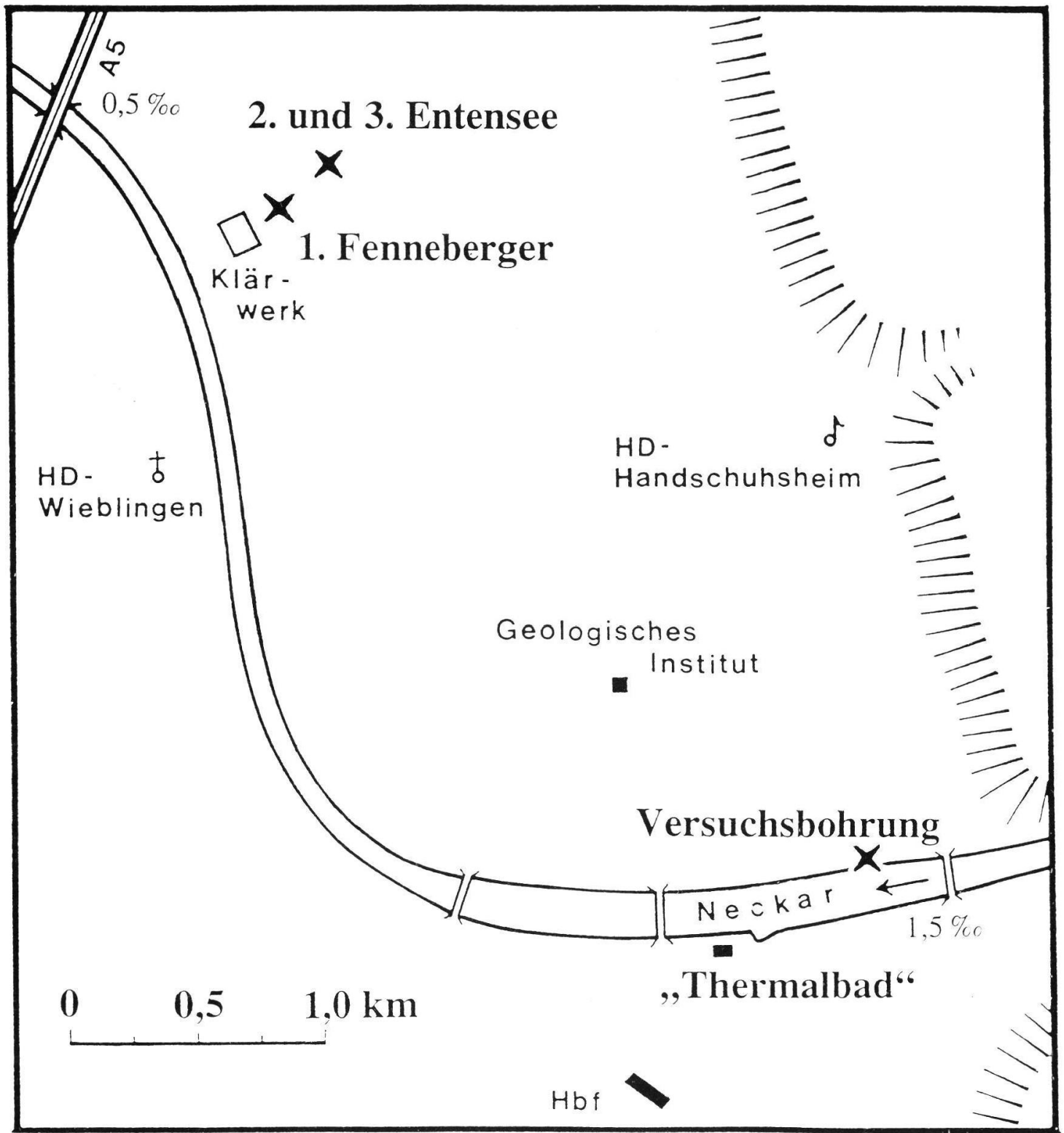

Abb. 2: Ausgewertete Bohrungen in Heidelberg.

Fig. 2: Situation of boreholes in Heidelberg.

jetzt ab verdient der Buntsandstein seinen Namen, wenn er im dunkelgrauen Sandton eingestreut ist. In diesem ältesten Abschnitt des Pleistozäns hat der Neckar sein Sediment kaum geändert, es ist nur ein wenig gröber geworden. Vielleicht hat er am Ende etwas häufiger Hochwasser geführt.

Das Universitätsarchiv verwahrt ein von BubNoff gezeichnetes Profil des Bereichs von 630 bis $413 \mathrm{~m}$ Teufe. Im Abstand von 11-14 m (im Mittel 12,2 m), selten $24 \mathrm{~m}$, sind dünne Kieslagen eingetragen. Wenn wir den Trend, daß der Neckar im Lauf seiner
Geschichte immer stärker sedimentiert hat, nach rückwärts extrapolieren, dürfen wir eine Rate von $0,12 \mathrm{~mm} / \mathrm{Jahr}$ ansetzen. Dann hätte ein Zyklus 101000 Jahre gedauert; im 100 000-Rhythmus schwankt die Exzentrizität der Erdbahn-Ellipse (IMBRIE 1985).

Eine kürzere Periode ergibt sich, wenn wir die Abstände der Schichtwechsel, die der Bohrmeister notiert hat, von „hart" über „weich" bis „hart" berechnen. Zwar streuen sie ein wenig, häufen sich aber deutlich bei $5,2 \mathrm{~m} \pm 20 \%$. In diesem etwas jüngeren 


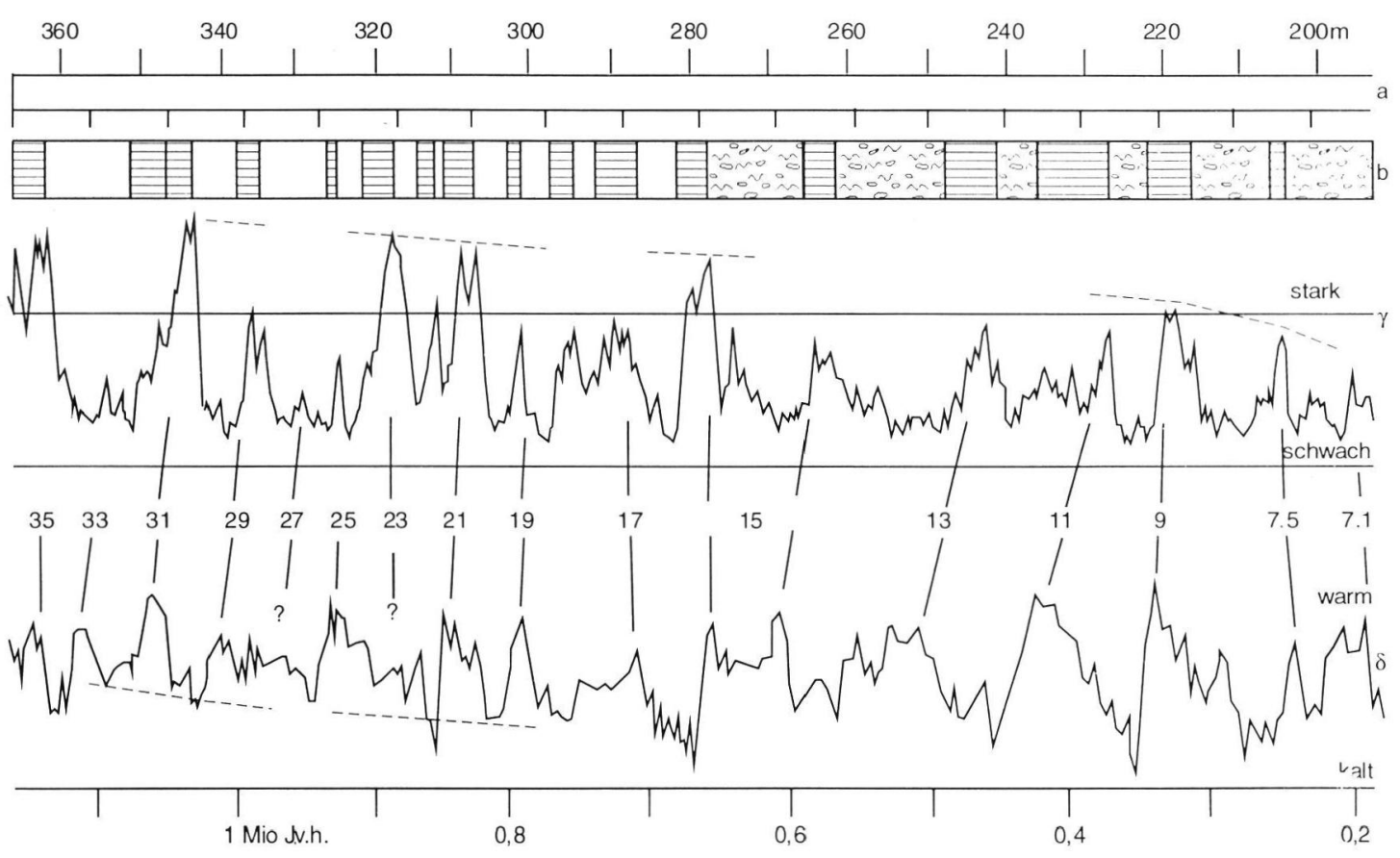

Abb. 3: Teile des Alt- und Mittelpleistozäns in der Versuchsbohrung „HI) 1 Fenneberger”. a) 'leufe in m, umgerechnet auf Profil „HD 3 Entensee“. b) Körnung. $\gamma$ = Gammastrahlung im Bohrloch (b und $\gamma$ aus SchneIDER 1975) Temperatur des Pazifiks vor der Küste von Ecuador (SHACKLETON 1989, Fig.5, Kern 677).

Fig. 3: Parts of Old and Middle Pleistocene in Heidelberg. a) Depth, b) grain size, $\gamma$ ) gamma radiation (= clay content), lower curve = oxygen isotopic record of planctic and benthic foraminifera in ODP site 677 off Ecuador (SHACKLETON 1989, fig.5). Lowest scale $=$ age B.P.

Bereich (500 bis $390 \mathrm{~m}$ Teufe) nähern wir die Sedimentation der im untersten Teil des Profils „HD 1 Fenneberger" ermittelten Rate an : 0,13 mm/J. Dann hätte ein Zyklus 40000 Jahre gedauert; das ist die Periode, mit der sich die Erdachse stärker neigt und wieder aufrichtet (ImBrIE 1985). Fällt eine 40 000Warmzeit auf eine 100 000-Kaltzeit, so wird jene unterdrückt. In Heidelberg wird kein Kies abgelagert, der Abstand wächst auf $24 \mathrm{~m}$. Die Beschreibung des Thermalprofils endet in derjenigen Teufe, ab welcher das Profil „HD 1 Fenneberger“ zur Verfügung steht.

\section{Das Profil „Heidelberg 1 Fenneberger“ Graubraune Sande von 365 bis 285 m Teufe}

Vier $\mathrm{km}$ nordwestlich von der Wurzel des Neckarschwemmfächers, nahe dem jetzigen Wasserwerk Entensee, wurde 1973 eine Versuchsbohrung auf sauberes Trinkwasser angesetzt, die $350 \mathrm{~m}$ Teufe erreichte. Aus den in Kernkisten ausliegenden Schichten wurden alle $2 \mathrm{~m}$ eine Probe ins geomorphologische Labor mitgenommen.

Über die verschiedenen Bohrverfahren, Analysenmethoden und über die Stratigraphie der jüngeren Schichten wurde früher berichtet (FEZER 1977, FEzER \& MeIer-Hilbert \& Schloss, 1992). Hier beschränke ich mich auf die Schichten zwischen 365 und $285 \mathrm{~m}$ Teufe. Die Werte wurden nachträglich auf die Skala der Bohrungen Entensee 2 und 3 umgerechnet, daher beginnt die Beschreibung nicht mit den tatsächlichen Ziffern der Sohle, sondern mit 365 m Teufe. Schon im Bohrloch hat die Fa. Tegtmeyer die Gammastrahlung gemessen und als Kurve aufgezeichnet (Abb. 3). Sie entspricht ungefähr dem Tonanteil, der z.T. direkt aus den Gesteinen und Böden des Keupers stammt oder durch Verwitterung anderer Gesteine entstanden ist. Nur dieser zweite Anteil hängt mit der Temperatur zusammen, indem in Warmzeiten einige Minerale schneller in Ton umgewandelt werden.

Nach einer Vergrößerung läßt sich die Heidelberger Gammakurve, also der Tongehalt, an die Temperaturkurve von SHACKLETON (1989) gemäß dem Tiefseekern 677, erbohrt vor Ecuador, anlegen. Mehrere Peaks wurden nach Form und Amplitude an ähnlichen Zacken der anderen Kurve festgehakt (Abb. 3). Jeder Peak muß nicht nur in die Reihenfolge passen, sondern die Kurven müssen auch ähnlich verlaufen. Die Zeit ist nach der etwas moderneren Berechnung von BASSINOT et al. (1994, 103) angegeben. Die Übereinstimmungen überwiegen, es gibt aber auch Unterschiede. Die Warmzeit 27 tritt im Pazifik 


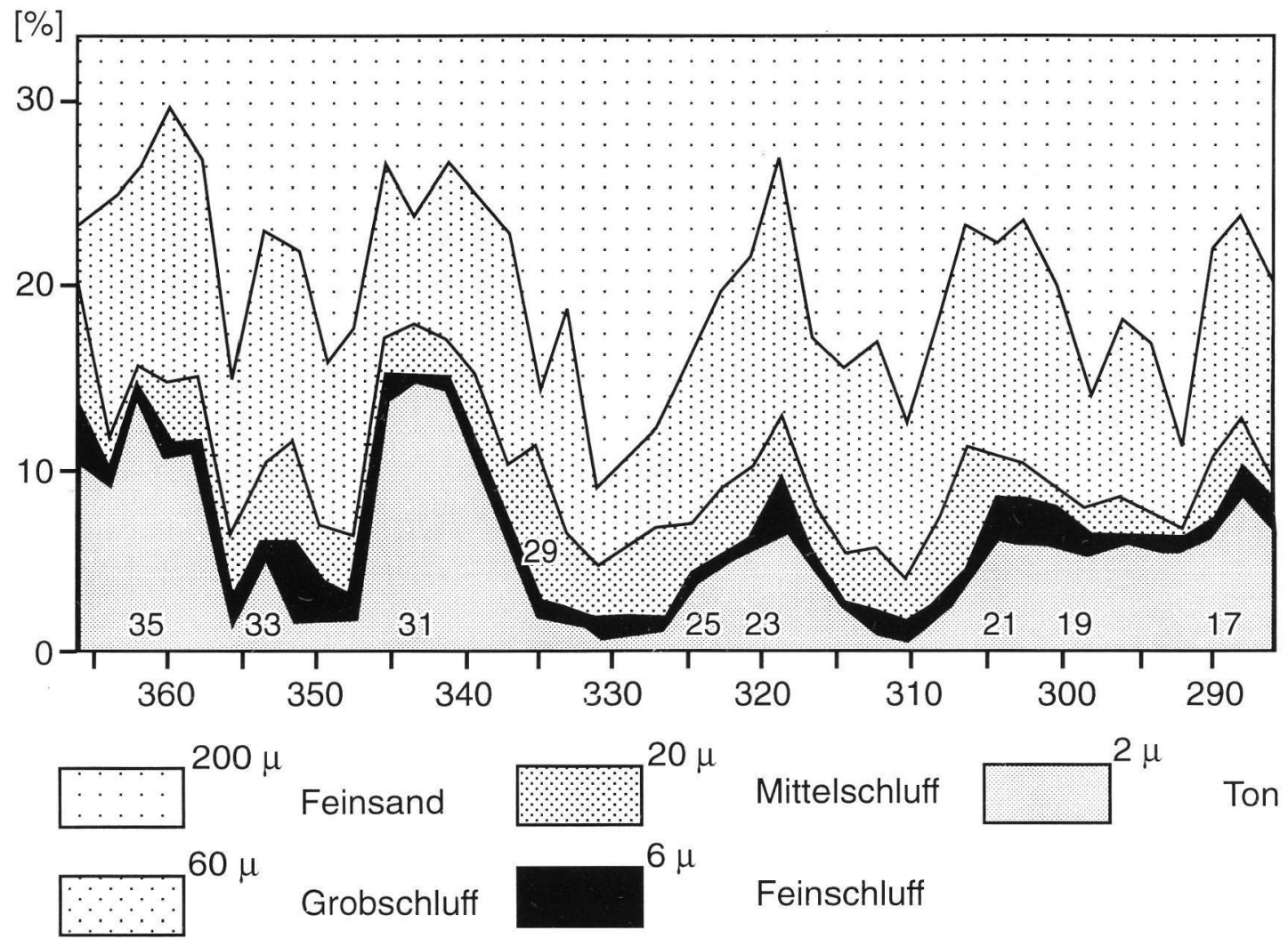

Abb. 4: Gliederung der Fraktion Feinsand, Schluff und Ton aus der Bohrung „HD 1 Fenneberger“. Sauerstoff-IsotopenStadien 35-17. (Entw. u. Zeichn. G. Koos 1975 unveröff.).

Fig. 4: Fine sand, silt and clay percentages in the borehole 1 . Numbers $=$ oxygen isotopic stages. Lowest line $=$ depth. Ton $=$ clay.

kaum heraus, im Heidelberger Gamma-log etwas deutlicher. Die Warmzeit 25 erscheint am Neckar kürzer als im Ozean, vielleicht 15000 Jahre. Die Warmzeit 23 gehört in Heidelberg zu den großen, in SHACKLETON's Kern 677 fehlt sie.

Für ein dünnes Sedimentpaket oder für eine einzelne Warmzeit ist es schwierig, Mächtigkeit und Zeitraum anzugeben, weil die Methode der Abgrenzung nicht genormt ist. Auf Werte, die von einem dicken Paket oder einer Gruppe von Kaltzeiten abgeleitet sind, können wir uns eher verlassen. Gehen wir auf Abb. 3 an den obersten Balken und greifen die ältesten 58 m Sediment ab, springen dann zur untersten Skala, so kommen wir auf 370000 Jahre. Der Neckar hat demnach in einem Jahr 0,15 mm sedimentiert. Eine einzelne Kalt- oder Warmzeit hat im Durchschnitt nur 23000 Jahre gedauert, viel kürzer als die späteren Perioden (Abb. 3).

Neben dem rhythmischen, kurzzeitigen Auf und $\mathrm{Ab}$ zeigt die Kurve auch eine langfristige Tendenz: die Tonmaxima werden allmählich geringer, das Klima also kühler. Etwas Ähnliches deuten Pollenanalysen aus den Niederlanden an (ZAGWIJN, viele Arb.); die thermophilen Pflanzenarten nehmen von Warmzeit zu Warmzeit ab. Zwei Hypothesen stehen zur Wahl. 1. Die Zwischenspeicher für tertiäre Böden, Hangund Flußsedimente werden Zug um Zug entleert.

2. Die Temperaturen sinken in einem großen Teil der Erde.

Die ozeanische ${ }^{180}$-Kurve spricht für die zweite Erklärung : Die Minima sinken von Kaltzeit zu Kaltzeit immer tiefer ab, dies ist auf Abb. 3 durch eine gestrichelte Trendlinie angedeutet. Die beiden Ausreißer, nämlich die Kaltzeiten 34 und 22 fallen im Neckarton nicht auf.

\section{Sedimentanalyse}

Koos (1975) hat die Bohrproben untersucht. Die meisten enthielten nur 1-3\% Kies. Weil uns die Zahl der Gerölle zu gering schien, wich er auf den groben Grobsand aus (2 - 1,25 mm Ø). Zu unserer Überraschung waren beide Fraktionen ähnlich zusammengesetzt. Die Gruppe Ton, Schluff und Feinsand nimmt 15-30\% ein. Der in Abb. 4 rasch erkennbare Tonanteil schwankt erheblich, tonarme Schichten sind reich an Sand. Die Tonpeaks lassen sich alle in der Gamma- 


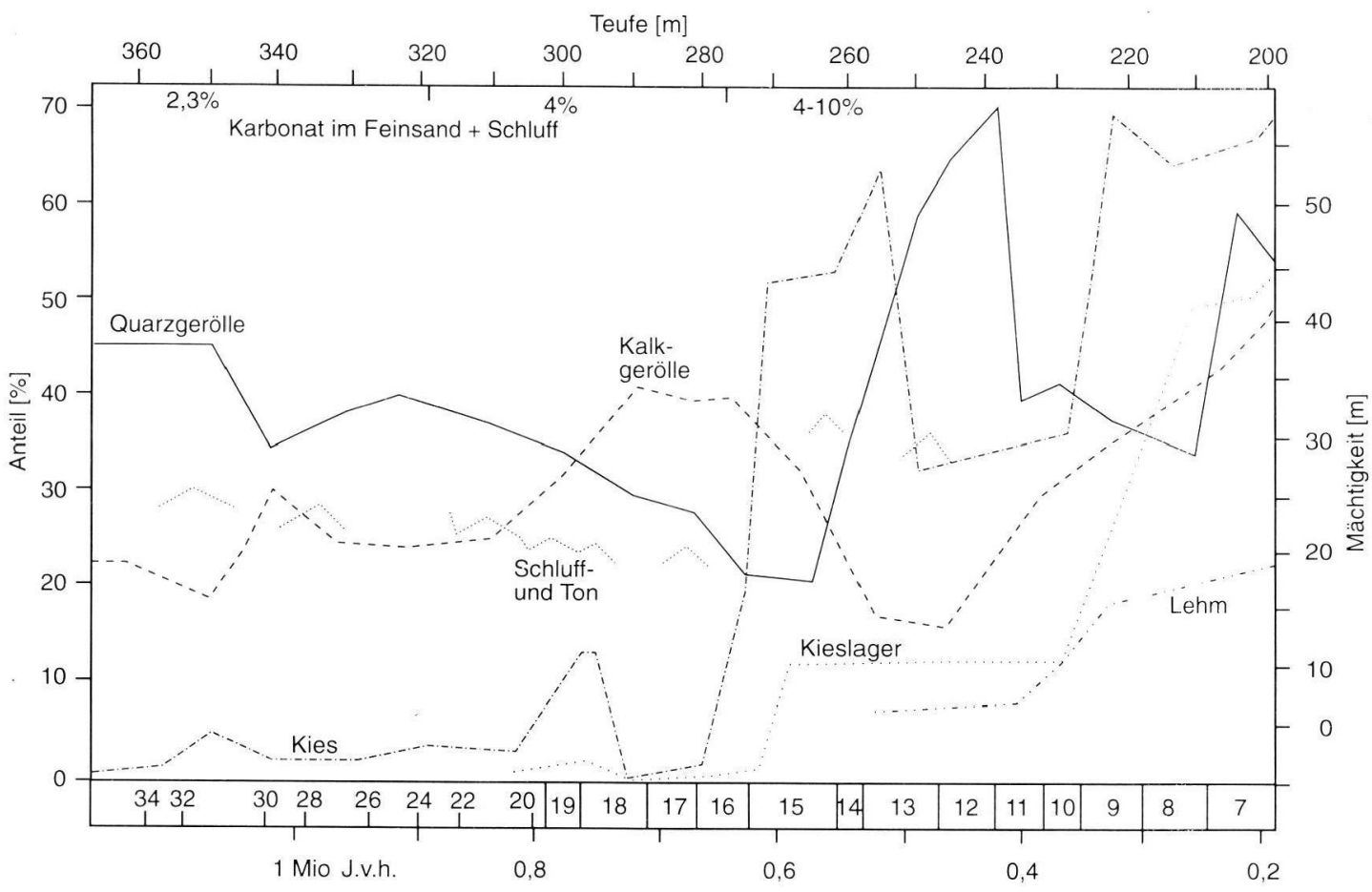

Abb. 5: Zusammensetzung alt- und mittelpleistozäner Neckarsedimente. Linke Skala = Kiesanteile $(>2 \mathrm{~mm} \emptyset)$ an mehreren Gesamtproben. „Quarz“- und „Kalk“-Anteile am Kies. Schluff + Ton = Anteile am Feinmaterial <0,2 mm $\varnothing$. Rechte Skala $=$ Schichtmächtigkeiten (sandige Kiese, Lehm $=$ Sand und Schluff. Unten $=$ Sauerstoff-Isotopen-Stadien im Ozean und Alter (Mio J.v.h.).

Fig. 5: Old and Middle Pleistocene sediments. Left scale $=$ percentage, above $=$ depth, right scale $=$ thickness, below $=180$-stages and time B.P. $($ Mio y.). Kalk = limestone, Kies $=$ gravel, Lehm $=$ loam, Schluff $=$ silt.

kurve (Abb. 3) wiederfinden (umgekehrt nicht alle) und mit den üblichen Ziffern bezeichnen.

In den Warmzeiten 29 und 27 war der Ozean nur mäßig warm, die Heidelberger Gammastrahlung mäßig bzw. schwach, und im Sediment schwindet der Tongehalt auf 2 und $0,5 \%$ des Feinmaterials. Die Tonkurve der Abb. 4 löst die Zeit schlechter auf, weil Koos nur alle $2 \mathrm{~m}$ eine Probe zur Verfügung hatte. Die scheinbar eintönige Folge von Sanden paßt gut zu dem nur mäßigen Wandel der Ökosysteme, wie er durch Pollenanalysen bekannt geworden ist. In diesen älteren Kaltzeiten wurde die Vegetation kaum geschädigt (LANG 1994, 271). Verglichen mit den älteren Schichten des Thermalprofils sind die Fenneberger Sedimente etwas gröber. Stets dominiert der Mittelsand, der 50-60\% einnimmt, der Grobsand 15-25\%, Feinsand 15-20\%, der Rest besteht aus Schluff und Ton. Bei der Betrachtung von Grobsand und Feinkies können wir die Schichten in drei Abteilungen gliedern.
Jede Gruppe endet mit einer tonarmen Schüttung: der Gehalt schrumpft fast auf Null (Abb. 4); dagegen wachsen Grobsand und Kies. Der grobe Grobsand (2 - 1,25 $\mathrm{mm} \varnothing)$ ist am Anfang stark ausgelesen, der Quarz nimmt 80-95 \% ein, ganz allmählich geht der Gehalt auf $60 \%$ zurück. Seine Stelle nehmen die Gerölle frischer Gesteine ein; z.B. wachsen im Feinkies

Buntsandstein von $5-10 \%$ auf $33 \%$ Muschel- und Jurakalk jeweils auf $\quad 20 \%$ In der Mittelsandfraktion nehmen die Schwermineralien jeweils

$$
\text { von ca. } 1 \% \quad \text { auf } \quad 4 \% \mathrm{zu} \text {. }
$$

BARTZ erwähnt aus $397 \mathrm{~m}$ Teufe der „Thermalbohrung“, was -332 m am Entensee entsprechen könnte, Gerölle aus Quarz, schwarzem Karneol, viel Kristallin, Quarzrhyolith und Hornstein.

Alle drei Schichten mit frischem Material zeichnen sich in der Gammastrahlung durch geringen Tongehalt aus, der Ozean war damals kühl (Abb. 3), aber nicht kälter als in anderen Kaltzeiten. Es zeigt sich auch kein mittelfristiger Trend der Minimal- oder Maximalwerte wie beim Grobsediment. So ist die Ursache der Dreigliederung eher im hydrologischen 


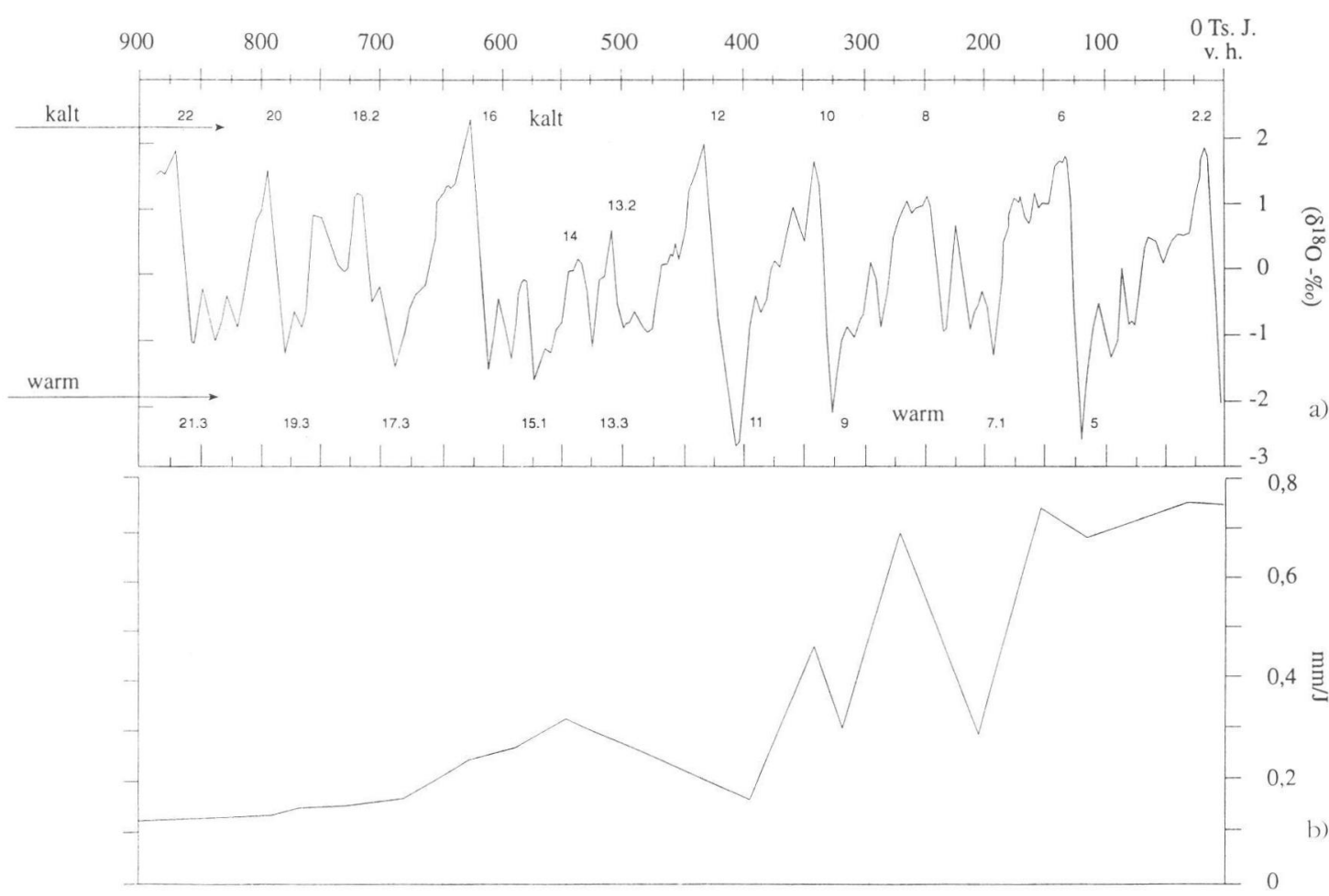

Abb. 6: a) 180-Proportionen (Temperatur tropischer Meere, kombiniert aus den Bohrkern 900963 vor den Malediven und 677 vor Ecuador (aus BAssinot et al. 1994, 103). b) Sedimentationsrate des Neckars in Heidelberg-Entensee.

Fig. 6: a) Oxygen isotopic temperature from Indic and Pacific cores. Mumber = stage (after Bassinot et al. 1994, 103).

b) Rate of sedimentation in Heidelberg-Entensee $(\mathrm{mm} /$ year).

als im klimatischen Wandel zu suchen. Während eines Schluff-Ton-Minimums von -320 bis $-318 \mathrm{~m}$ (Kaltzeit 24) springt im Feinsand der Karbonatgehalt aufs Doppelte und bleibt so noch lange Zeit bis -290 m (Kaltzeit 18). Vermutlich wurde das Klima etwas kühler, das Sediment wurde rascher durchtransportiert und verwitterte schwächer.

Wichtiger sind die langfristigen Wandlungen. Sie werden sichtbar, wenn wir Maxima, Mittelwerte oder Minima einzelner Indikatoren auf den Kurven verbinden (Abb. 5). Zu Anfang des Pleistozäns sprechen hohe Quarz- und Tongehalte für eine intensive Verwitterung (Abb. 3 und 4). Sie nehmen ganz allmählich ab. Langsam werden die Flußsedimente gröber (Abb. 5), und im Feinkies nehmen die Kalkgerölle schubweise zu. Bei -340 m gehen die Quarzgerölle von 45 auf $35 \%$ zurück, dagegen wachsen Weißjura und Muschelkalk von ca. 20 auf $30 \%$. Beides spricht für eine lebhaftere Erosion und relativ raschen Transport. Auch der geringe Schluffanteil deutet auf eine schwache Verwitterung in einem kühlen Klima.

\section{Neckarsande in Heidelberg (300 bis $285 \mathrm{~m}$ Teufe) und Mauer (132 bis $141 \mathrm{~m} \mathrm{NN}$ ), Kaltzeit 18 und Warmzeit 17}

Weil sich diese Schichten mit den jüngst erbohrten Sanden im Liegenden der homo-Fundstelle korrelie- ren lassen (Fezer \& Meier-HilberT \& Schloss 1992), sollen sie eingehend beschrieben werden. In dieser Zeit ändert sich das Sediment: der Gehalt an Ton, Schluff und Feinsand schwindet von 30 auf $17 \%$, statt dessen wächst der Grobsand auf $35 \%$. Neuartig ist, daß Gerölle, die in den älteren Kaltzeiten nur einzeln eingestreut waren, bei $-298 \mathrm{~m}$, an einem der mittelfristigen Grenzpunkte, in einem kurzen Schwall $13 \%$ einnehmen. Anfangs bestehen 35-55\% davon aus Quarz; der Neckar hat ältere Restschotter durch Seitenerosion mitgerissen. Wie in jüngeren Kaltzeiten schrumpft der Anteil allmählich auf $30 \%$; Quarz wird z.T. durch frischen Muschelkalk ersetzt (wächst von 6 auf $22 \%$ ). Die Wärmezeiger Schluff und Ton sowie die Quarzgerölle nehmen ab, die Kältezeiger Kiesanteil und Kalkgehalt nehmen zu. Was diese Gerölllage heraushebt, ist ein erster Schub von weißen, plattigen Geröllen : 16 - 23\% Malmkalk von der Schwäbischen Alb, welche damals noch weiter in Richtung Stuttgart gereicht hat.

10 km südöstlich von Heidelberg, am Rand des Dorfes Mauer, liegt die ehemalige Sandgrube Grafenrain, in welcher 1907 der Unterkiefer des „homo erectus heidelbergensis" gefunden worden ist. Von der Sohle aus wurde 1991 zweimal bis auf den anstehenden Muschelkalk gebohrt (Zöller \& STREMmE 1992, 96 in Beinhauer \& Wagner). Über dem Fels la- 
gert eine Schicht, die dem Profil „Heidelberg 1 Fenneberger" im Bereich -300 $\mathrm{m}$ ähnelt.

Im Bohrprofil Mauer hat URBAN (1992, 117 in BEINHAUER \& WAGNER) zwischen 134,2 und 137,3 m NN (das könnte am Entensee den Teufen 299 bis 293 entsprechen) Pollen gefunden. Ein „Wald mit taigaartigen Florenelementen" weicht in einem kühlen Stadial (18.2 ?) Gräsern und Kräutern, später kommen wieder Birken und Kiefern, schließlich Fichten hinzu.

Wie in Heidelberg besteht der Kiesanteil zu 17-38\% aus Malmkalk, $30 \%$ Muschelkalk und 20-50\% Buntsandstein (Zählung Dr. M. Löscher). Weil Warmzeiten allmählich in Kaltzeiten übergehen und sich nicht eindeutig abgrenzen lassen, wurden die Stadien 18-16 zusammengenommen, um die Sedimentationsrate abzuschätzen :

$\begin{array}{ll}\text { Mauer } & 0,075 \mathrm{~mm} / \mathrm{Jahr} \\ \text { Heidelberg } & 0,135 \mathrm{~mm} / \mathrm{Jahr}\end{array}$

Später, in der Zeit des homo erectus heidelbergensis, hat der Neckar in Mauer langsamer, in Heidelberg schneller sedimentiert.

Der chaotische Verlauf der Kurven in der rechten Hälfte der Abb. 5 ist indirekt vom Klima ausgelöst. Entweder im Stadium 20 oder in 18 hat die von Tübingen kommende Ur-Lone (Gg. WAGNeR 1963) ihr flaches Tal so hoch aufgeschottert, daß sie bei Plochingen in Richtung Stuttgart überlief. Dadurch geriet das Geosystem aus den Fugen. Zuerst wurden alte, quarzreiche Schotter mitgerissen, dann erodierten die Flüsse in die Tiefe und brachten Kalkgerölle. Reste dieser Massen blieben in Obrigheim-Mörtelstein, Mauer und Heidelberg erhalten (Fezer 1992, 99, in Beinhauer \& Wagner).

Im Teufenbereich von -292 bis - $285 \mathrm{~m}$ ist der Mittelsand tonhaltig. Die Fraktionsgruppe FeinsandSchluff-Ton enthält plötzlich nur noch halb so viel Kalk, der Mittelsand doppelt so viele Schwermineralien. Bei den Geröllen setzt der Malmkalk aus, der Quarzanteil steigt von 22 auf $33 \%$. Diese Schicht stammt also aus einer Warmzeit. Dies ist in Mauer noch deutlicher. Zwischen 138 und $141 \mathrm{~m} \mathrm{NN}$ lagert Ton, der sekundär entkalkt ist; der Rest besteht aus Illit, Smektit und Kaolinit (Schweizer \& Menges \& Christmann 1992, 149, in Beinhauer \&Wagner).

\section{Ergebnisse und Ausblick}

Flußsedimente, auch solche weit ab von Gletschern, spiegeln den Klimawandel. Sie lassen sich einfacher mit den Sauerstoff-Isotopen-Kurven der Ozeane und der großen Eisschilde korrelieren als Gruppen von Moränen oder glazifluvialen Schottern. Im Altpleistozän zeigt ein starker Gamma-log und ein hoher Quarzgehalt eine Warmzeit an.

Langfristiges Verhalten von Klima-Indikatoren im Altpleistozän:
Ton $>$ Quarz $>$ Kalk $<$ Feldspat $<$ Korn- $\varnothing<$ Aus vegetationskundlicher Sicht läßt LANG (1994, 271) mit dem Stadium 19 das Altquartär enden. Vorher, in den kurzen Kaltzeiten, wurden thermophile Arten nur wenige $100 \mathrm{~km}$ nach Süden abgedrängt und konnten in der nächsten Warmzeit rasch und ohne Verluste wieder zurückwandern.

Im Ozean ähneln die 180-Kurven in den Stadien 18 bis 13 teils dem älteren, teils dem jüngeren Typ. Die Kaltzeit 16 dauert bereits recht lang, das Wasser kühlt tiefer aus als vorher; ab jetzt werden die Temperaturkurven asymmetrisch. Der Neckar verhielt sich bis zur Warmzeit 17 brav und lagerte nur $0,15 \mathrm{~mm}$ Sediment/Jahr ab, vom Stadium 16 bis 14 waren es 0,28. Er bringt in der Warmzeit 15.5 ein letztes Mal viel Ton. Zwischen den Stadien 19 und 15 ändert sich also der Charakter der Kaltzeiten. Nach dem Verlauf der 180Kurve (Abb. 3) möchte ich das Altpleistozän erst um 2 Stadien später als Lavg enden lassen und die Grenze zwischen den Stadien 17 und 16, also bei 700000 J.v.h. ziehen.

Die spätere Entwicklung wurde in anderen Publikationen (Fezer \& Meier \& Schloss 1992, Fezer 1998) eingehend beschrieben. Die Schotterpakete werden mächtiger (statt 3-7 m später 16-20 m, schließlich $60 \mathrm{~m}$ ), teils weil die Kalt- und Warmzeiten länger dauern, teils weil der Neckar jährlich 2-3 mal so viel Sand ablagert. Aus der Zusammensetzung der Feinund Mittelkies-Fraktion lassen sich die Prozesse aufhellen. Im Mittelpleistozän sind einzelne Kaltzeiten so intensiv, wie wir es von den beiden jüngsten kennen. Dazwischen ereignen sich auch solche, die nur mäßig kalt waren, in denen die Gletscher nicht aus Skandinavien und den Alpen herausgekommen sind und deren Spuren später weggewischt wurden.

Die Neckarsedimente in der Nähe des Wasserwerks Entensee haben das Klima des Jungtertiärs und des gesamten Quartärs mit ganz geringen Schichtlücken überliefert. Eine Forschungsbohrung mit anschließender interdisziplinärer Auswertung würde sich lohnen.

\section{Dank}

Der Bohrfirma Göttker und den Herren SchNeIDER sei Dank für ihre Hilfe bei der Probenahme. Besonders zu danken ist Herrn Gunter KOOS für die sorgfältige Analyse der Sedimente.

\section{Schriftenverzeichnis}

BARTz, J. (1951): Revision des Bohrprofils der Heidelberger RadiumSol-Therme. Jber.Mitt.oberrhein.geol.Ver. NF 33: 101-125, 2 Abb., Freiburg.

Bassinot, F. C. \& Labeyrie, L. D. \& Vincent, E. \& Quidelleur, X \& Shackleton, N. J. \& Lancelot, Y. (1994): The astronomical theory of climate and the age of the Brunhes-Matuyama magnetic reversal. Earth \& Planetary Sci. 126: 91-108, 7 Abb., 4 Tab., Amsterdem. 
Beinhauer, K. W. \& WAGner, G. A. (Hrsg., 1992): Schichten von Mauer, 85 Jahre Homo erectus heidelbergensis. Mannheim-Heidelberg (Braus), 192 S., 167 Abb.

Fezrr, F. (1974): Randflußs und Neckarschwemmfächer. Heidelberger Geograph.Arb. 40: 167-183, 9 Abb., 1 Tab., Heidelberg. - (1977): Analysis of River Sediments and Quaternary Ecology. Catena 4: 135-138, 2 Abb., Gießen.

- (1993/95): Nikr contra Elsa, zur Flußgeschichte des unteren Neckars. HGG-Journal 7/8: 30-38, 6 Abb., 2 Tab., Heidelberg. - (1998): Mittel- und Jungpleistozän im „Heidelberger Loch“ Bohrprofil Entensee von 285 bis 6 m Teufe. Jber. Mitt. + oberrhein. geol. Ver. NF 80, 17 Abb., 17 Tab.

Fezer, F. \& Meier-Hiıbert, G. \& Schloss, S. (1992): Vergleich der Mauerer Sande mit den datierten Bohrprofilen aus dem Heidelberger Neckarschwemmfächer. Jber. Mitt. oberrhein. geol. Ver. NF 74: 149-161, 7 Abb., 2 Tab., Stuttgart.

Hsieh, J. C. C. \& Murray, B. (1996): A 24000 year period climate signal in 1.7 - 2.0 million year old Death Valley strata. Earth \& Planet, Sci. L. 141: 11-19, 3 Abb., Amsterdam.

Imbrie, J. (1985): A theoretical framework for the Pleistocene ice ages. J. Geol 142: 417-432, 19 Abb., 1 Tab., London.

Koos, G. (1975): Gliederungsversuch für das Quartär in der Trinkwasserbohrung Heidelberg-Fenneberger, Teil 3: 270-350 m Teufe. Hausarb. wiss. Prüf. f. d. L., Geograph. Inst. Heidelberg, $36 \mathrm{~S}$. (masch.schr.) $15 \mathrm{Abb}$.
LANG, G. (1994): Quartäre Vegetationsgeschichte Europas. 462 S. 177 Abb., 54 Tab., Jena.

MADER, M. (1978): Die Flußgeschichte des Neckars und das Wandern des Albtraufs. Veröff. Naturschutz Landschaftspfl. BadenW. 47/48: 443-507, 7 Abb., 3 Tab., Karlsruhe.

Salomon, W. (1927): Die Erbohrung der Heidelberger Radium-SolTherme und ihre geologischen Verhältnisse. Abh. Heidelb. Akad. Wiss., math. nat. Kl. 14: 1-105, 5 Abb., 36 Tab., Heidelberg.

SCHLÜCHTER, C. (1992): Terrestrial Quaternary Stratigraphy. Quarternary Sci. Rev. 11: 603-607, 2 Abb., (Pergamon)

SCHNEIDER, E. \& H. (1975): Synsedimentäre Bruchtektonik im Pleistozän des Oberrheintalgrabens zwischen Speyer, Worms, Haardt und Odenwald. Münster. Forsch. Geol. Paläont. 36: 81126, 15 Tafeln, Münster i.W.

Shackieton, N. J. (1989): The Plio-Pleistocene ocean, stable isotope history. In: J. ROSE \& C. SCHLL̈CHTER (Hrsg.): Quarternary type sections, imagination or reality ? 11-24, 8 Abb., Rotterdam

Manuskript eingegangen am 06.01.1997 